\title{
Modeling Foamy Oil Flow in Porous Media
}

\author{
D.D. Joseph ${ }^{1}$, A.M. Kamp ${ }^{2}$, R. Bai ${ }^{1}$ \\ ${ }^{1}$ Univ. of Minnesota, Dept. of Aerospace Engng. \& Mech, 107 Akerman Hall \\ 110 Union Street. S.E., Minneapolis, MN 55455, USA \\ ${ }^{2}$ PDVSA Intevep, PO Box 76343, Caracas 1070-A, Venezuela
}

October 2001

\begin{abstract}
Certain heavy oils which foam under severe depressurization give rise to increased recovery factor and an increased rate of production under solution gas drive. These oils not only stabilize foam, but also stabilize dispersion of gas bubbles at lower volume ratios. The way this phenomenon is related to the chemistry of the oil and its viscosity is presently not understood. We present here a mathematical model of reservoir flow of foamy oil which depends only on the velocity through Darcy's law, the pressure and the dispersed gas fraction. The theory governs only in situations in which the bubbles do not coalesce to produce the percolation of free gas. In this theory the bubbles move with the oil as they evolve. The main empirical content of the theory enters through the derivation of solubility isotherms which can be obtained from PVT data; modeling of nucleation, coalescence, bubble drag laws and transfer functions are avoided. The local pressure difference and dispersed gas fraction are in equilibrium on the solubility isotherm. In a pressure drawdown the time taken for the system to return to equilibrium is described by a rate law characterized by an empirical relaxation time (rate constant). The resulting systems of equations can be reduced to a coupled pair of nonlinear PDE's for the dispersed gas fraction and pressure difference, which can further be reduced in the equilibrium case to a second order evolution equation for the pressure difference. This system of equations can also be derived from usual theory of two-phase flow in a porous media based on relative permeability under the assumption that the bubbles and oil move in lock step. We propose a reformulation of the conventional theory in which the concept relative permeability of the porous media is replaced with the more familiar concept of an effective phase viscosity. The equations of our relaxation theory are solved numerically, and the mixture viscosity function and relaxation time are selected to match the sandpack experiments of Maini and Sarma [1994].
\end{abstract}




\section{Introduction}

In this paper a model is presented that is motivated by the need to explain anomalous features associated with production from reservoirs of so-called foamy oils. These oils are described by some of their properties of response to pressure declines; it is noted that they nucleate dispersed gas bubbles and display obvious foaminess in well head samples produced by solution gas drive in which oil and gas are produced by the drawdown of pressure (Huerta, Otero, Rico, Jiménez, De Mirabal, \& Rojas, [1996], De Mirabal, Gordillo, Fuenmayor, Rojas, Rodriguez \& Sanchez, [1996]).

When compared with the response of conventional oils, the response of foamy oils to drawdown of pressure is more favorable; primary recovery factor (percentage of the oil in the reservoir which can be recovered), the rate of production, the volume ratio of oil to gas which is recovered and the length of time that a given pressure gradient or rate of production can be maintained are all increased substantially; the reasons for the favorable response of foamy oils in solution gas drive are not well understood and tentative explanations which have been put forward are controversial (see Maini [1996], Pooladi-Darvish and Firoozabadi [1997] and Sheng, Maini, Hayes \& Tortike, [1999a] for recent reviews).

Foamy oils carry considerable amounts of dissolved gases in the condensed state. The relevant thermodynamic property for this is "gas solubility"; a function of temperature and pressure at equilibrium which gives the volume ratio of dispersed gas from the crude oil by outgassing. Tables of solubility of methane, carbon dioxide and other gases in various Canadian crude oils have been given by Svrcek \& Mehrotra [1982], Peng, Fu, Bird, \& Hsi [1991] and others. The oils considered to be foamy evidently cavitate small dispersed bubbles which, under some conditions, are believed to move with the crude oil in which they are dispersed. Experiments done by Pooladi-Darvish \& Firoozabadi [1997] have shown that bubbles which arise from depressurization of silicone oil and heavy crude of equivalent viscosity are very different; the bubbles in the silicone oil are larger and much more mobile than the ones in crude oil. Viscosity alone might not be enough to demobilize dispersed gas; it is desirable to look at other properties like surface tension and surface active agents. Possibly there are surface active agents which are present naturally in foamy crudes which allow them to stabilize foam, but the precise agents, their composition and the mechanics by which they are released apparently have not been studied.

A "foamy oil" is a heavy oil which foams under rapid depressurization. Maini [1996] notes that ". . . the term "foamy oil" is often used to describe certain oils produced by solution gas drive which display obvious foaminess in wellhead samples. The primary production of heavy oil from several reservoirs in western Canada is 
in the form of an oil continuous foam. This foam resembles a chocolate mousse in appearance and often persists in open vessels for several hours..." Foaming at a well head is a kind of foam formation analogous to the head on beer. To get such a head the pressure decline must be sufficiently severe to allow the bubbles which rise from outgassing of condensed gas in the bulk to accumulate at the free surface faster than they collapse. Perhaps there are surfactants in foamy oil which stabilize the films between the bubbles preventing collapse, promoting the buildup of the head. Foam stability measurements in the laboratory have shown that the foaminess of crude heavy oils is comparable to aqueous foams used for steam flooding applications (Sheng, Hayes, Maini \& Tortike [1996]). The outgassing of condensed gas will not lead to foam at the well head if the rate of depressurization is too low.

To create foam in a reservoir or in a sandpack it is necessary to depressurize rapidly enough to produce close packed solution gas bubbles which can undergo a topological phase change to stable films and plateau borders. This kind of in situ foaming of sandpacks has been achieved in the experiments of Maini \& Sarma [1994]. Gas and liquid move in lock step in these foams and lead to very high primary recovery factors. At the actual reservoir, gas fractions can be as low as 5 to $10 \%$. At these gas fractions the oils cannot foam but are well-dispersed and protected against coalescence, possibly by the same natural surfactants that might stabilize the foams.

In solution gas drive of foamy oil the depressurization of the sample leads to cavitation of small dispersed bubbles. The volume ratio of dispersed gas increases the volume of our composite fluid and it acts as a pump, gas coming out of solution pumps the fluid outward. This pumping action is well described by the continuity equation (4.4) which implies that in a closed volume $v$ with boundary $S$ containing dispersed bubbles of volume fraction $\phi$

$$
\int_{v} \frac{1}{1-\phi} \frac{D \phi}{D t} d v=\oint_{S} \mathbf{u} . \mathbf{n} d S
$$

where $\mathbf{n}$ is the outward normal on $S$ and $\mathbf{u}$ is the velocity of our composite fluid.

The relative velocity of dispersed gas is important; if the bubbles coalesce and move relative to the oil more gas and less oil will be produced. Good recovery is sometimes described by a critical gas saturation value; this is the volume fraction of gas at which the gas becomes connected and starts to move faster than the oil. Maini [1996] identifies this critical saturation as a percolation limit, whilst Firoozabadi, Ottensen, Mikkelsen and December [1992] and Pooladi-Darvish and Firoozabadi [1997] identify this even by visual observation of bubbles in a viewing window. The values given by Firoozabadi et al. are about 5 times smaller than those given by Maini and his coworkers. 
Mastmann, Moustakis and Bennion (2001) wrote a paper "Predicting foamy oil recovery" which they applied to the Driza crude oils found in a large Albanian oil field. This oil gives rise to huge amounts of stable foam under rapid depressurization. They say

The basic mechanism of foamy oil behavior is related to the existence of what is commonly termed a "pseudo" bubble point. The true bubble point is the pressure at which, at the reservoir temperature, the first small bubbles of free gas evolve from solution in the oil and nucleate as a distinct free gas phase. For most conventional oils having relatively low viscosity, the gas rapidly coalesces into large bubbles and evolves almost immediately from the oil to form a separate and distinct gas phase. In many reservoirs, this forms a secondary gascap during depletion operations and results in high gas-oil ratios at production wells due to the high mobility of a free gas phase in the porous media in comparison to the more viscous oil. For this reason, for conventional or non-foamy oils, the bubble point and the "pseudo" bubble point have the same or very close to the same values.

For foamy oils, due to the high viscosity of the oil, the gas bubbles cannot immediately coalesce together to form bubbles large enough to allow gravitational forces to separate from the oil. For this reason, the oil phase remains as a continuous dispersed gas-oil emulsion with a higher and higher concentration of increasingly larger bubbles trapped in a milkshake-like format within the oil as the pressure is reduced. The point for the foamy oil at which the bubbles of free gas can finally start to escape from solution as a distinct free gas phase is known as the pseudo bubble point.

Their use of the term "pseudo bubble point" is slightly unconventional. We prefer to refer to this condition as a "percolation threshold." This threshold is a fluid mechanics, possibly rheological property of the oil and the producing reservoir or sand pack, and not a thermodynamic property; it could depend on time. Under very rapid depressurization in a sandpack or at the well head in the reservoir, the gas is nucleated so rapidly that it closes packs and, in a foamy oil, forms a foam without gas percolation. In a reservoir, after long time evolution of gas the mechanics of coalescence is such as to lead to percolation.

When the gas percolates, the good news about recovery is over; it is no wonder that all authors find that the critical saturation values are about the same as the primary recovery factors (which is the fraction of oil recovered by solution gas to oil in the reservoir) even when they disagree about definitions. 
A few models of foamy oil flow have been put forward; each emphasize some special feature. One of the most recent models by Sheng et al. [1996], Sheng et al. [1999a] are multiphase theories based on conservation laws with transfer from solution gas to dispersed gas and from dispersed gas to free gas. Their theory does not seem to follow the curve of experimental values. Sheng et al. [1996] say that

Published models include the psuedo-bubble point model (Kraus, McCaffrey, \& Boyd [1993]), the modified fractional flow model (Lebel [1994]) and the reduced oil viscosity model (Claridge \& Prats [1995]). Maini [1996] gave a detailed review and discussion of some of these models. These models have been used to history match heavy oil production, but their common weakness is that the dynamic processes which are important features of foamy oil flow were not included properly. Although it may be possible to get an acceptable history match using these models, the predictive ability is likely to be limited...

A more successful approach to modeling was recently advanced by Sheng, Hayes, Maini, \& Tortike [1999b]. This model also requires the modeling of nucleation, bubble growth and disengagement of gas bubbles from the oil. The model ultimately leaves undetermined two adjustable parameters which fit the theory to experimental data better than previous models.

To predict foamy oil behavior, Mastmann et al. (2001) use "Eclipse" which is a commercial "black oil" simulator. The model equations at the foundation of this simulator are the conventional multiphase ones based on the concept of relative permeability (see section 2) which is not well suited to the description of a homogenous mixture in which the oil and gas move in lock step. Obviously, in such a state the molecular viscosity of the gas is not important. The "black oil" model allows for gas evolution through solubility tables and not through an equation of state like our (2.14)

The present theory could be called a continuum mixture theory which is appropriate for foamy oil flow with dispersed gas of low mobility relative to the liquid and leads to three coupled nonlinear partial differential equations for $\mathbf{u}, p$ and the gas fraction $\phi$, five scalar equations in five unknowns. Our model has a few features in common with the excellent early work of Leibenson [1941] on the motion of gas saturated fluid in a porous media.

The model proposed here does not require information about nucleation, bubble growth, compressibility or forces which produce relative velocity. We put up a one-phase or mixture theory in which the dispersed gas is described by a gas fraction field in a single fluid in which the viscosity, density and mobility in Darcy's law all depend on the gas fraction. This fluid satisfies the usual Darcy law, and the continuity equation together with a kinetic (constitutive) equation required by 
the condensation and outgassing of methane (or other gases) in heavy crude. The theory depends only on parameters which can be measured in a PVT cell and sandpack. The virtue of the model is simplicity, but it can work only for relatively immobile dispersed gas bubbles in which divergence-free velocities are excluded (see the discussion following (4.4)). Certainly such a theory could not be expected to give rise to a percolation threshold or even to a critical gas fraction. We shall show that it can describe many features of solution gas drive of foamy oils in the regimes when the bubbles in the mixture are dispersed and even when they are trapped in foam.

The assumption that bubbles move in lock step with the oil could not hold when the diameter of a large number of the nucleated bubbles grow larger than a typical pore diameter. Nor would our theory work if the diameter of many of the bubbles were so small as to produce a sensible effect on the mixture pressure. Under the high pressure conditions of a typical reservoir one atmosphere is very small and even a one micron bubble with a tension of 30 dynes/cm would produce a capillary pressure of 6 atmospheres. Our theory then assumes a large fraction of small bubbles but is not restricted to small gas fractions.

It is our idea that the increased recovery and production are generated by the pumping of nucleating and growing gas bubbles embodied in (1.1). However recovery factors and production rates are not the same and we might test some ideas: if two foamy oils have the same viscosity, the one with higher solubility having more gas to release will have higher primary recovery and production rate; if two foamy oils have the same solubility, the one with lower viscosity will have a higher rate of production because the mixture is more mobile, but a lower primary recovery because the percolation threshold is lower. If the oil foams in situ, oil and gas move in lock step and the primary recovery factor increases while due to the increased viscosity of foam the rate of production could decrease.

\section{Equation of state for dispersed gas}

Solubility isotherms are an equation of state for foamy oil in which all of the gas not in solution is dispersed in small bubbles. Free connected gas is not allowed. When the pressure is dropped, the mass of dissolved gas released goes entirely into dispersed gas bubbles. We have obtained solubility isotherms from measurement in PVT cells and applied the results to characterize the outgassing and absorbing of gasses in foamy oil in porous media. We have adopted the view that foamy oil will have the same equation of state in a porous media as a PVT cell; the thermodynamics of the fluid are independent of the wall of the containing solid provided that these walls are not so closely spaced as to effect the thermodynamic properties 
of the bulk fluid.

In the experiments of Svrcek \& Mehrotra [1982] the pressure is dropped from $p$ and $T$ to $p_{\text {ref }}$ and $T_{\text {ref }}$ where in the experiments $p_{\text {ref }}$ is atmospheric and $T_{\text {ref }}=$ $373.2^{\circ} \mathrm{K}$. It is assumed that all the gas in the live oil at $p, T$ comes out. Defining now:

$V_{g}(p, T)$ is the volume of dispersed gas,

$V_{l}(p, T)$ is the volume of live oil,

$V^{*}(p, T)$ is the volume of dispersed gas which evolves from the condensed gas when $p, T$ are dropped to $p_{\text {ref }}, T_{\text {ref }}$.

In this model we avoid all constitutive equations regarding nucleation rates and bubble growth. In our model we have only a mixture of liquid and dispersed gas, and the dispersed gas enters only through the volume fraction

$$
\phi=\frac{V_{g}}{V_{l}+V_{g}} .
$$

Equation (2.1) may be solved for

$$
V_{g}=\frac{\phi V_{l}}{1-\phi}
$$

Svrcek and Mehrotra [1982] give volumetric solubility curves $\left(\mathrm{CO}_{2}\right.$ and methane, figures 8 and 12, pages 34 and 36). Their results show that

$$
\hat{V}=V^{*} / V_{l}(p, T)
$$

is a linear function of pressure, with a larger slope at lower temperatures. We can assume that this tells you how much dispersed gas can come out of a solution of dissolved gas in oil which is at a saturation value at any pressure and temperature. We are going to assume that this $\hat{V}$ determines the dispersed gas fraction $\phi$ following an argument put forward in what is to follow.

The data of Svrcek and Mehrotra [1982] show that

$$
p-p_{\text {ref }}=\hat{\gamma}(T) \hat{V}
$$

where $\hat{\gamma}=d p / d \hat{V}$ is the slope of the solubility isotherms shown in figures 8 and 12. This slope is approximately constant. Here we have chosen $p_{\text {ref }}$ as a small pressure at which a negligible amount of gas is dissolved in the oil. For practical purposes this could be standard (atmospheric) pressure. In the present approach we have no way to predict the size distribution of gas bubbles. This means that we 
are free to choose the size and distribution to measure $\hat{V}$ and the most convenient choice is when all the released gas is collected at the top of a PVT such as in the experiment of Svrcek \& Mehrotra.

We first suppose that all the gas which comes out of a solution is dispersed and does not percolate or foam. We must suppose that the nucleation, growth and compressibility of gas bubbles are working, but these microstructural features are not monitored in these experiments which give only the solubility $\hat{V}$. This is also what we do in the mathematical model.

To convert (2.4) into a relation between $p$ and $\phi$ at equilibrium we note that the total mass $M$ of gas in the live oil is invariant, independent of $p$ and $T$ and

$$
M=M_{g}(p, T)+M_{c}(p, T)
$$

where

$M_{g}(p, T)$ is the mass of dispersed gas.

$M_{c}(p, T)$ is the mass of condensed gas.

Since the mass of condensed gas does not change when it is vaporized and assuming that this vapor is a perfect gas, we have

$$
M_{c}=p_{\mathrm{ref}} V^{*} / R T_{\mathrm{ref}}
$$

where $R$ is the gas constant. From the same gas law

$$
M_{g}=p V_{g} / R T
$$

Hence, from (2.5), (2.6) and (2.7) we have

$$
M=\frac{p V_{g}}{R T}+\frac{p_{\mathrm{ref}} V^{*}}{R T_{\mathrm{ref}}}
$$

and, using (2.3) and (2.4)

$$
M=\frac{p V_{g}}{R T}+\frac{p_{\text {ref }}}{R T_{\text {ref }}}\left(\frac{p-p_{\text {ref }}}{\hat{\gamma}}\right) V_{l} .
$$

We next introduce the bubble point pressure $\tilde{p}$ as the pressure at which there is no dispersed gas, all the gas is dissolved in the live oil so that $V_{g}=0 ; \tilde{V} \stackrel{\text { def }}{=} \hat{V}$ when $V_{g}=0$ and from (2.4)

$$
\tilde{p}-p_{\text {ref }}=\hat{\gamma}(T) \tilde{V}
$$


where

$$
\tilde{V}(\tilde{p}, T)=V^{*}(\tilde{p}, T) / V_{l}(\tilde{p}, T) .
$$

Since $M$ is invariant, we may evaluate (2.8) at the bubble point

$$
M=\frac{p_{\text {ref }}}{R T_{\text {ref }}} V_{l}(\tilde{p}, T) \tilde{V}(\tilde{p}, T) .
$$

Using (2.10) to eliminate $\tilde{V}$ in (2.12) and equating (2.12) and (2.9) we get

$$
\frac{T_{\text {ref }}}{T} \frac{p}{p_{\text {ref }}} V_{g}+\left(\frac{p-p_{\text {ref }}}{\hat{\gamma}}\right) V_{l}(p, T)=\left(\frac{\tilde{p}-p_{\text {ref }}}{\hat{\gamma}}\right) V_{l}(\tilde{p}, T) .
$$

In most depressurization experiments the change of liquid volume due to outgassing and compressibility is small and $V_{l}(p, T) \approx V_{l}(\tilde{p}, T)$. In this case the terms proportional to $p_{\text {ref }}$ in (2.13) subtract out and after replacing $V_{g}$ with $\phi V_{l} /(1-$ $\phi)$ we get

$$
\beta \frac{\phi}{1-\phi}=\frac{\tilde{p}-p}{p}
$$

where

$$
\beta=\frac{T_{\text {ref }}}{T} \frac{\hat{\gamma}}{p_{\text {ref }}}
$$

is completely determined by the solubility isotherm. Since the variation of $T$ is small on an absolute scale, the values $T / T$ ref are just slightly larger than one.

The variable $\hat{V}$ is called gas-oil ratio and $\tilde{V}$ is the gas-oil ratio at saturation pressure. From (2.10):

$$
\hat{\gamma}(T)=\frac{\tilde{p}-p_{\text {ref }}}{\tilde{V}}
$$

Substituting this value of $\hat{\gamma}$ in (2.15)

$$
\beta=\frac{T_{\text {ref }}}{T} \frac{\tilde{p}-p_{\text {ref }}}{p_{\text {ref }} \tilde{V}}
$$

In most practical situations $\tilde{p} \gg p_{\text {ref }}$ so that from (2.17)

$$
\beta=\frac{T_{\text {ref }}}{T} \frac{\tilde{p}}{p_{\text {ref }} \tilde{V}} .
$$

It is also customary in the oil industry to characterize live oil by its saturation pressure $\tilde{p}$ and its gas-oil ratio $\tilde{V}$ at saturation pressure. By virtue of (2.18) one can 
calculate the solubility parameter $\beta$. Note that in most experiments $p_{\text {ref }}$ is chosen as atmospheric pressure and $T_{\text {ref }}$ as $60^{\circ} \mathrm{F}=15.6^{\circ} \mathrm{C}$.

Values for $\beta$ for two Canadian heavy oils, Lloydminster and Lindbergh (Maini $\&$ Sarma, 1994) and for a Venezuelan heavy oil, Cerro Negro, are given in table 2.1. Graphs of the isotherm (2.14) for various values of $\beta$ are shown in figure 2.1.

It can be noted that the solubility value for heavy crude oils from very different regions are very close, which indicated that they contain similar amounts of dissolved gases at the same pressures. The oil industry calls oil with dissolved gas "live oil."

When $\phi$ and $p$ satisfying (2.14) vary from point to point

$$
\nabla p=\frac{-\beta p^{2}}{\tilde{p}(1-\phi)^{2}} \nabla \phi
$$

According to Darcy's law, $\mathbf{u}=-\lambda \nabla p$ where $\lambda$ is the mobility of the foamy mixture in the porous media; hence the fluid flows up the bubble gradient toward regions in which there are more bubbles where the pressure is smaller.

Departures from the equilibrium solubility relation (2.14) are indicated as $s u$ persaturation or subsaturation. Defining the function

$$
f(p, \phi) \stackrel{\text { def }}{=} \tilde{p}-p-\beta p \phi /(1-\phi),
$$

supersaturation $(f>0)$ corresponds to having more gas dissolved than there should be under thermodynamic equilibrium; subsaturation $(f<0)$ corresponds to having less gas dissolved than there should be under equilibrium. Supersaturation occurs when the pressure in the reservoir is drawn down, but the oil cannot evolve gas fast enough to keep up with the depressurization. Subsaturation, on the other hand, occurs when there is not enough gas available to dissolve in order to satisfy thermodynamic equilibrium at prevailing reservoir pressure and temperature. The function $f$ in our theory is thus an indicator for departure from equilibrium solubility.

\begin{tabular}{lll}
\hline Oil & $\beta$ & $\mathrm{T}\left({ }^{\circ} \mathrm{K}\right)$ \\
\hline Lloydminster & 3.40 & 293 \\
Lindbergh & 3.17 & 293 \\
Cerro Negro & 3.53 & 327 \\
\hline
\end{tabular}

Table 2.1: Solubility coefficients for some heavy oils. 


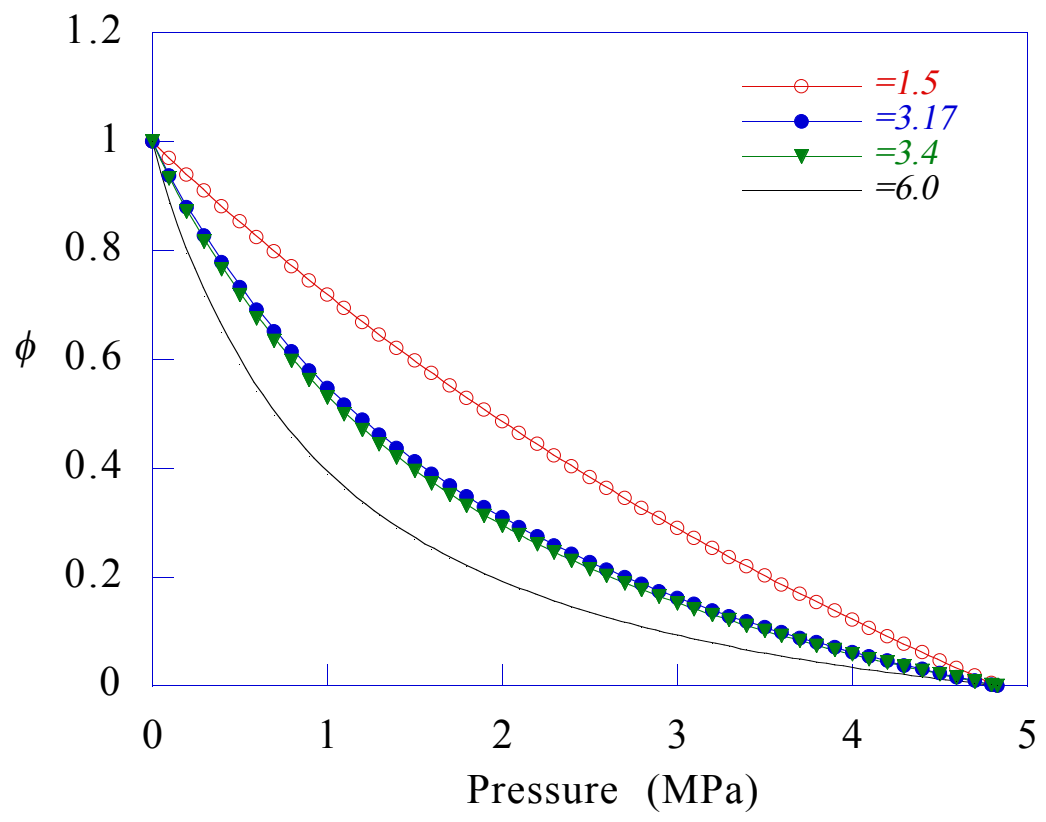

Figure 2.1: Graphs of the solubility isotherm (2.14) for various values of $\beta$. The limits of validity of the dispersed gas model can be roughly set at a close packing value, say $\phi_{c}=0.68$. When $\phi>\phi_{c}$ some bubbles must touch and form foam film or to coalesce. This implies that results for drawdown greater than those for which $\phi=0.68$ must take into account foaming and fingering of free gas.

The equilibrium isotherm $f=0$ allows for dispersed gas fraction $\phi$ from zero to one; from all liquid to superdry foam. Our theory makes no distinction between bubbly mixtures, wet or dry foams.

Consider a drawdown to atmospheric pressure $p_{a}=10^{6}$ dynes $/ \mathrm{cm}^{2}$ from a saturation pressure $\tilde{p}=4.83 \times 10^{7}$ dynes $/ \mathrm{cm}^{2}$ as in the experiment of Maini \& Sarma [1994]. Using $\beta=3.4$ for Lloydminster (from table 2.1) and evaluating the gas fraction of $\phi=\phi_{a}$ at atmospheric pressure we find

$$
\phi_{a}=0.93 \text {. }
$$

This is too much gas to exist as a bubbly dispersion; either the bubbly mixture passed into foam near to some critical value, say

$$
\phi=\phi_{c}=0.680
$$

or close packing or else some of the bubbles coalesced as free gas which fingers out 
of the sandpack. Probably foam and free gas both evolve at the outlet of a closed sandpack after a sudden drawdown to atmospheric from high saturation.

We also note that the physics of supersaturation is complicated by the fact that bubbles cannot nucleate continuously and that the mechanism by which they nucleate is still not well understood. A bubble which might form in crude oil by the vaporization of dissolved gas at supersaturated conditions can be expected to satisfy Laplace's law

$$
p_{\text {vapor }}-p=2 \gamma / R
$$

where $\gamma$ is surface tension (say 30 dynes $/ \mathrm{cm}$ ) and $R$ is the bubble radius. Under mildly supersaturated conditions $p$ is slightly smaller than the vapor pressure $p_{\text {vapor }}$; hence, $R$ satisfying (2.23) cannot be very small. In deriving (2.14) we have assumed the continuity of pressure across the bubble surface, ignoring the small pressure drop implied by (2.23).

It is argued that the vaporization of dissolved gas under supercritical conditions requires the simultaneous presence of undissolved gas hidden in crevices of impurities which are wet by gas preferentially. The curvature of the gas-oil interface in such a crevice is opposite to a bubble and it is controlled by capillarity rather than interfacial tension. The supersaturated dissolved gas vaporizes at the undissolved gas hidden in the crevice and the volume of the gas grows there until a bubble breaks away restoring the nucleation site to its original condition. The train of gas bubbles which emanate usually from a single site on a glass of beer as the gas comes out of the solution is a convenient example of outgassing at a nucleation site. The pore walls in a porous media are nucleation sites for outgassing of foamy oil. The possibility that asphaltenes in the oil are nucleation sites for dissolved gas is a current but unresolved question.

\section{Live oil and dead oil}

Oil without dissolved gas is called dead oil. Oil saturated with dissolved gas is called live oil. The dissolved gas can be considered condensed and live oil is a mixture of miscible liquids, dead crude oil and condensed gas. The mixture of miscible liquids is like glycerin and water with the caveat that the oil and dissolved gas do not mix in all proportions; the fraction of dissolved gas at saturation is a function of temperature and pressure. The weight fraction of dissolved gases range from 0.3 to $6 \%$. (See the tables corresponding to figures 8 and 12 in Svrcek \& Mehrotra [1982]).

The viscosity of live oil can be orders of magnitude smaller than the viscosity of dead oil; the viscosity of live oil is a strongly decreasing function of the amount 
of dissolved gas in solution just as the viscosity of glycerol strongly decreases with the water fraction. In a pressure decline the viscosity of the live oil will increase because less gas is dissolved and because the presence of dispersed gas should increase the viscosity of the composite fluid.

We may seek to answer the question "what is the density of the dissolved gas in solution." We are not able to measure the density of the dissolved gas in oil, but the density $\rho$ of the mixture is available in data presented by Svrcek and Mehrotra (1982, see figures 6 and 11, pages 34 and 36). Let us note that this data shows that the density of the $\mathrm{CO}_{2}$ in bitumen is a weak function of the pressure; hence the density of the non-saturated bitumen is independent of the volume ratio $\Psi$ of soluble gas in bitumen. Dissolved methane has a density only slightly different than bitumen (see their figures 8 and 12). A theory of miscible mixtures which applies to live oil can be found in Chapter X of Joseph \& Renardy [1992].

\section{Model description}

In this model we avoid all constitutive equations regarding nucleation rates and bubble growth. In our model we have only foamy oil and dispersed gas and the dispersed gas enters only through its volume ratio $\phi$. The model combines Darcy's law, with a $\phi$ dependent mobility, a mass conservation law for ideal mixtures together with a constitutive equation governing the evolution of departures from equilibrium solubility. For flow not in equilibrium gradients and time derivatives are crucial. The time derivatives which are used here have a material derivative

$$
\frac{D}{D t}=\alpha \frac{\partial}{\partial t}+\mathbf{u} \cdot \nabla
$$

where $\alpha$ is the porosity. The continuity equation is given by

$$
\frac{d \rho}{d \phi} \frac{D \phi}{D t}+\rho(\phi) \operatorname{div} \mathbf{u}=0
$$

where

$$
\rho(\phi)=\rho_{g} \phi+\rho_{l}(1-\phi) \approx \rho_{l}(1-\phi)
$$

because $\rho_{g} \ll \rho_{l}$ where $\rho_{l}$ is the density of live oil which depends only weakly on the volume ratio of dissolved gas. Combining (4.3) and (4.2) we find that

$$
\frac{D \log (1-\phi)}{D t}+\operatorname{div} \mathbf{u}=0
$$

Equation (4.4) restricts the theory to dispersions of low mobility relative to the suspending liquid. In any motion $\mathbf{u}(\mathbf{x}, t)$ of the composite which is divergence free 
$\operatorname{div} \mathbf{u}=0$, the dispersed gas fraction does not change following the motion of the fluid

$$
\frac{D \phi}{D t}=\frac{\partial \psi}{\partial t}+\mathbf{u} \cdot \Delta \psi=0 .
$$

Bubbles rising under gravity would lead to divergence free motions as would any motion of the bubbly mixture in which dispersed bubbles do not nucleate, diffuse or compress. Motions with non-zero divergence satisfy (1.1); the flux out of any closed volume, over which the div $\mathbf{u}$ does not sum to zero, must be non zero. This is the simplified way that our theory accounts for nucleation and diffusion.

Turning next to Darcy's law, we let $x$ increase in the direction of gravity. Then

$$
\mathbf{u}=-\lambda\left\{\nabla p-\rho g \mathbf{e}_{x}\right\} \approx-\lambda\left\{\nabla p-\rho_{l} g(1-\phi) \mathbf{e}_{x}\right\}
$$

where

$$
\lambda(\phi)=k / \mu(\phi)
$$

is the mobility, $\mu(\phi)$ is the viscosity of live oil with dispersed gas of volume ratio $\phi$ and $k(\phi)$ is the permeability. The viscosity $\mu(\phi)$ increases with $\phi$ for two reasons: dispersed gas bubbles and the reduction of live oil viscosity due to outgassing of light components.

\subsection{Relative permeability}

Equations (4.2), (4.5) and (4.6) are implied by the usual theory for two phase flow through porous media based on relative permeabilities under the assumption that the average liquid and gas velocities are equal. The demonstration of this fact leads also to a formula (4.24) for ratio of the relative permeabilities under the same assumption.

The composite volume average velocity is given by

$$
\mathbf{u}=\mathbf{U}_{l}+\mathbf{U}_{g}
$$

where

$$
\mathbf{U}_{l}=(1-\phi) \mathbf{u}_{l}, \mathbf{U}_{g}=\phi \mathbf{u}_{g}
$$

are superficial velocities (volume flow rate over total area) and $\mathbf{U}_{l}$ is the average oil velocity and $\mathbf{U}_{g}$ is the average gas velocity. The mixture density $\rho(\phi)$ is given by (4.3) and the composite mass average velocity is given by

$$
\mathbf{u}_{m}=\frac{\left[\rho_{l}(1-\phi) \mathbf{u}_{l}+\rho_{g} \phi \mathbf{u}_{g}\right]}{\rho(\phi)} .
$$


The equations expressing the conservation of mass of each of the two phases are

$$
\begin{gathered}
\alpha \frac{\partial \rho_{l}}{\partial t}(1-\phi)+\nabla \cdot \rho_{l}(1-\phi) \mathbf{u}_{l}=0 \\
\alpha \frac{\partial \rho_{g} \phi}{\partial t}+\nabla \cdot \rho_{g} \phi \mathbf{u}_{g}=0 .
\end{gathered}
$$

When $\mathbf{u}_{l}=\mathbf{u}_{g}$, the average gas and liquid velocities are equal and from (4.7), (4.8) and (4.9)

$$
\mathbf{u}_{m}=\mathbf{u}=\mathbf{u}_{l}=\mathbf{u}_{g}
$$

After adding (4.10) and (4.11), using (4.12) we get equation (4.2).

We now introduce relative permeability and show that we may replace the concept of relative permeability with an equivalent concept of effective viscosity, which is a more familiar concept in the general theory of mixtures. Neglecting gravity and capillarity we have

$$
\begin{gathered}
(1-\phi) \mathbf{u}_{l}=\mathbf{U}_{l}=-\frac{k k_{r l}}{\eta_{l}} \nabla p=-\frac{k}{\mu_{l}(\phi)} \nabla p \\
\phi \mathbf{u}_{g}=\mathbf{U}_{g}=-\frac{k k_{r g}}{\eta_{g}} \nabla p=-\frac{k}{\mu_{g}(\phi)} \nabla p
\end{gathered}
$$

where $k$ is the permeability which we will identify as the $k$ in (4.6), $k_{r l}$ and $k_{r g}$ are relative permeabilities, $\eta_{l}$ and $\eta_{g}$ are molecular viscosities and

$$
\mu_{l} \stackrel{\text { def }}{=} \frac{\eta_{l}}{k_{r l}} \quad, \quad \mu_{g} \stackrel{\text { def }}{=} \frac{\eta_{g}}{k_{r g}}
$$

are effective viscosities.

The introduction of effective viscosities is nothing more than a definition; however, the definition implies that the differential flow resistance can be associated with two fluid phases and not with the porous media. Noting now that $\mathbf{u}$ in (4.5) is the composite velocity, we may write (4.5) and (4.6) neglecting gravity as

$$
\mathbf{u}=-\frac{k}{\mu(\phi)} \nabla p
$$

and using (4.7) together with (4.13) and (4.14), we get

$$
\frac{1}{\mu(\phi)}=\frac{1}{\mu_{l}(\phi)}+\frac{1}{\mu_{g}(\phi)} \text {. }
$$


Equation (4.17) and (4.15) relate the effective viscosity of the gas-liquid mixture to the relative permeabilities.

The consequences of our basic assumption that the dispersed gas moves with the liquid $\mathbf{u}=\mathbf{u}_{g}=\mathbf{u}_{l}$ may be extracted from the above equations. We find that

$$
\frac{1}{\mu_{l}(\phi)}=\frac{1-\phi}{\mu(\phi)}
$$

and

$$
\frac{1}{\mu_{g}(\phi)}=\frac{\phi}{\mu(\phi)}
$$

The effective gas and liquid viscosities are completely determined by the effective viscosity of the mixture when $\mathbf{u}=\mathbf{u}_{g}=\mathbf{u}_{l}$. Moreover, the ratio of relative permeabilities is

$$
\frac{k_{r l}}{k_{r g}}=\frac{\eta_{l} \mu_{g}(\phi)}{\eta_{g} \mu_{l}(\phi)}=\frac{1-\phi}{\phi} \frac{\eta_{l}}{\eta_{g}}
$$

is determined by $\phi$ and the ratio of molecular viscosities.

When $u_{g} \neq u_{l}$, we may introduce

$$
\chi=u_{l} / u_{g}
$$

and then

$$
\frac{k_{r l}}{k_{r g}}=\frac{\eta_{l} \mu_{g}(\phi)}{\eta_{g} \mu_{l}(\phi)}=\frac{(1-\phi) \eta_{l} \chi}{\phi \eta_{g}}
$$

The ratio $\chi$ of local velocities is related to the ratio of superficial velocities by

$$
\chi=\frac{U_{l} \phi}{U_{g}(1-\phi)}
$$

$\chi$ is a useful and easily computed parameter for flow diagnosis. When $\chi=1$, the flow is homogeneous. When $\chi=0$, the flow is all gas and the gas has percolated.

When $\chi \neq 1$, the homogeneous theory leading to the continuity equation (4.2) does not apply. Instead, the conservation equations (4.10) and (4.11) for the gas and liquid phase must be used. 


\subsection{Constitutive equations relating the dispersed gas fraction to the pressure}

We are proposing models in which the basic variables are the pressure and dispersed gas fraction and are such that disturbed systems which are not forced will relax to equilibrium with pressure and temperature on the solubility isotherm $f(p, \phi)=0$ given by (2.14). There are many possible ways to build models with the above properties. The simplest conceptual model with the desired properties is a first evolution model

$$
\tau \frac{D f}{D t}=-f
$$

which guarantees that a disturbed stationary system will relax exponentially. The implied assumption that both factors in the equilibrium isotherm relax at exactly the same rate is probably overly restrictive.

The constitutive equation

$$
\tau_{1} \frac{D}{D t}\left(\frac{\phi}{1-\phi}\right)+\tau_{2} \frac{D}{D t}\left(\frac{\tilde{p}-p}{p}\right)=\frac{\tilde{p}-p}{p}-\frac{\beta \phi}{1-\phi}
$$

allows for separate rates of relaxation. Obviously this equation is very closely tied to the equilibrium isotherm. To evaluate the possibility that a simple model based on (4.24), involving only the pressure and dispersed gas fraction, can describe solution gas drive of foamy oil, we must integrate the equations and apply the results to experiments and field operations. In this work, we apply our model to the experiments of Maini \& Sarma [1994]. The model requires that we identify a viscosity function $\eta(\phi)$ of the dispersed gas fraction and two relaxation times $\tau_{1}$ and $\tau_{2}$. In our first attempt at evaluation we put $\tau_{1}=0$; in this case (4.24) reduces to a Maxwell-like constitutive equation with pressure relaxation leading a hyperbolic system of PDE's that support wave propagation of pressure and bubble fronts. This system could not be made to simultaneously match experiments on steady flow, the rate of production of oil in the same steady flow, and the cumulative production in a blowdown experiment. We get a better match when $\tau_{2}=0$ with

$\tau_{1} \neq 0$ and we are able to select a viscosity function $\eta(\phi)=(1-\phi)^{-11}$ and $\tau_{1}$ to match all the sandpack experiments, but the match of model to drawdown experiments (figures 9.2 and 9.3) are less good. We speculate that the mismatch is due to percolation of free gas from the sandpack due to the massive pressure decline in blowdown that must, at best, lead to the copious formation of relatively dry foam.

Obviously we may get a better agreement with the model by fitting two relaxation parameters $\left(\tau_{1}\right.$ and $\left.\tau_{2}\right)$ rather than one; in this case we might expect to model 
short time phenomenon associated with nucleation. We prefer to proceed in the future by keeping fitting to a minimum and to look carefully at field data, not only the experiment of Maini \& Sarma.

In this work we confine our attention to the cases in which all the gas that comes out of a solution is dispersed in gas bubbles. In a pressure drawdown the gas bubbles nucleate and grow until the gas fraction $\phi$ is in equilibrium with the pressure, $f(p, \phi)=0$. We shall describe relaxation to equilibrium with a first order rate equation of the form (4.24) with $\tau_{2}=0, \tau_{1}=\tau$ written as

$$
\frac{\tau p}{(1-\phi)^{2}} \frac{D \phi}{D t}=f(p, \phi) \text {. }
$$

$D / D t$ is the usual expression (4.1) for the substantial derivative in a porous media. It is easy to verify that (4.24) and (4.25) give rise to relaxation. If the pressure is dropped from saturation, at which $p=\tilde{p}$ and $\phi$ to $p_{1}$, the initial response is given by

$$
\tau \frac{D(\phi / 1-\phi)}{D t}=\frac{\tilde{p}-p_{i}}{p_{i}}>0
$$

to that the dispersed gas will begin to increase toward the value of $\phi$ on the isotherm $f\left(p_{i}, \phi\right)=0$.

After eliminating $\mathbf{u}$ in (4.4) and (4.24) using (4.5), we find that

$$
\begin{gathered}
\tau\left(\alpha \frac{\partial}{\partial t}-\lambda \nabla p \cdot \nabla+\lambda \rho_{l} g(1-\phi) \frac{\partial}{\partial x}\right) \frac{\phi}{1-\phi}=\frac{\tilde{p}-p}{p}-\beta \frac{\phi}{1-\phi}, \\
\frac{1}{1-\phi}\left\{\alpha \frac{\partial \phi}{\partial t}-\lambda \nabla p \cdot \nabla \phi\right\} \\
=-\operatorname{div}(\lambda \nabla p)-2 \lambda \rho_{l} g \frac{\partial \phi}{\partial x}+\lambda^{\prime} \rho_{l} g(1-\phi) \frac{\partial \phi}{\partial x} .
\end{gathered}
$$

A mathematical theory for the system (4.27), (4.28) is not yet available. We note that except for $\operatorname{div} \lambda \nabla p$ the system is of first order, so that we might look at the problem as initial value problem for a second order PDE in $p$ for which end conditions on the pressure and initial conditions on the pressure and gas fraction are appropriate. We will solve these equations for sandpack problems in the sequel.

The system 4.24 and 4.5 may be regarded as describing the flow of a relaxing compressible fluid through a porous media. To see this, we replace $\phi$ with $\rho=$ 
$\rho_{l}(1-\phi)$, using 4.3. Then $4.24,4.4$ and 4.5 may be written as

$$
\left.\begin{array}{rl}
\tau\left[\alpha \frac{\partial}{\partial t}+\mathbf{u} \cdot \nabla\right] \frac{\rho_{l}-\rho}{\rho} & =\tilde{p}-p-\beta p \frac{\rho_{l}-\rho}{\rho}, \\
\alpha \frac{\partial \rho}{\partial t}+\mathbf{u} \cdot \nabla \rho+\rho \operatorname{div} \mathbf{u} & =0 \\
\mathbf{u} & =-\lambda \nabla p+\lambda g \rho \mathbf{e}_{x} .
\end{array}\right\}
$$

\section{Constant state solutions}

\subsection{Constant state solutions, drainage and stability}

The constant state solution are uniform solutions $p_{0}, \phi_{0}, \mathbf{u}_{0}$ of (4.27), $p_{0}$ and $\phi_{0}$ satisfying

$$
f\left(p_{0}, \phi_{0}\right)=\tilde{p}-p_{0}-\beta p_{0} \phi_{0} /\left(1-\phi_{0}\right)=0
$$

and

$$
\mathbf{u}_{0}=\mathbf{e}_{x} u_{0}, \quad u_{0}=\lambda\left(\phi_{0}\right) \rho_{l} g\left(1-\phi_{0}\right)
$$

The constant state solution is a drainage flow; this flow may be used to determine the mobility $\lambda(\phi)$. This solution is stable to small perturbations using normal modes (see Joseph, Kamp \& Bai [2002])

\section{Sandpack experiments}

Sandpack experiments are used as laboratory surrogates for the flow of oil and gas in porous reservoirs. In figure 6.1 a cartoon of a typical sandpack experiment copied from a paper by Sheng et al. [1996] is displayed. The pack may be loaded with sand from reservoirs.

Sandpacks provide an excellent way to get precise data under controlled conditions simulating flow in reservoirs; they are convenient for mathematical modeling because they lend themselves to one dimensional treatments. It is useful to look at these one-dimensional models for horizontal sandpacks in which gravity may be neglected and for vertical flow in which gravity may be important. In both these cases we have our governing equation (4.28) with $\nabla=e_{x} \partial / \partial x$. Terms proportional to gravity are put to zero in horizontal sandpacks.

Different experiments can be carried out in a sandpack corresponding to different conditions listed below. 


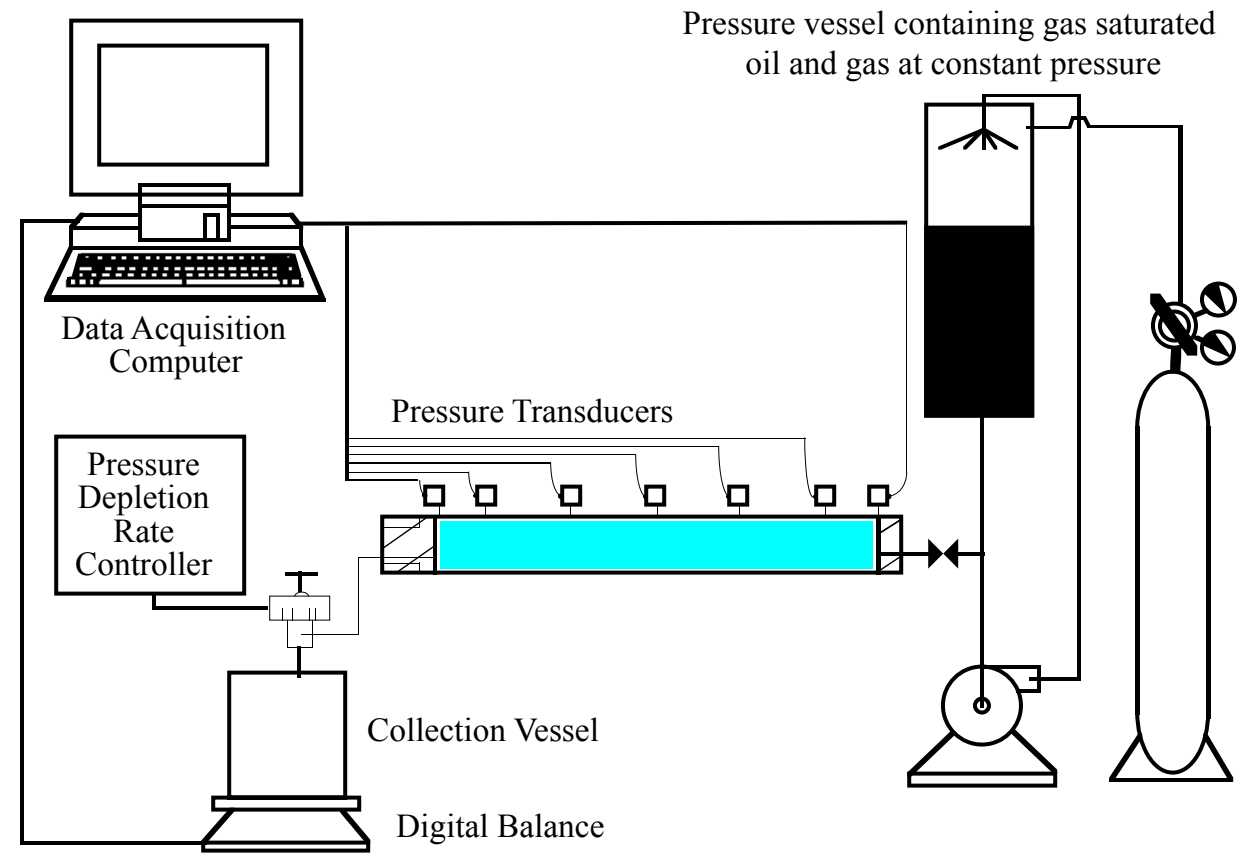

Figure 6.1: (Sheng, Hayes, Maini \& Tortike [1996].) Cartoon of a sandpack experiment. Live oil can be injected at the inlet and a pressure depletion rate controller is at the outlet. Different experiments are described by prescribed conditions at the inlet and outlet.

Maini \& Sarma [1994] reported results of experiments in a horizontal sandpack like that shown in figure 6.1 where pressure at inlet and outlet are controlled. A table of properties of the sandpack is given in table 6.2. "Prior to the start of the flow experiments, each oil sample was cleaned of its suspended materials. The oil was then recombined with methane gas in the recombination equipment at a pressure of 4.83MPa." They did steady flow experiments using Lloydminster and Lindbergh crude oil in which the inlet pressure was at saturation

$$
p=\tilde{p}=4.83 \mathrm{MPa}=4.83 \times 10^{7} \mathrm{dynes} / \mathrm{cm}^{2}
$$

varying the "drawdown" pressure at the outlet.

Table 6.3 gives the properties of the two "live" oils at saturation.

The pressure distribution along the core sample which was measured in steady flow by Maini and Sarma [1994] is shown in figure 6.2 and 6.3. Six pressure 


\begin{tabular}{lc}
\hline Parameter & Value \\
\hline Length $(\mathrm{m})$ & 2.0 \\
Cross-sectional area $\left(\mathrm{m}^{2}\right)$ & $16.1 \times 10^{-4}$ \\
Sand size $(\mu \mathrm{m})$ & $74-105$ \\
Porosity (fraction) & 0.33 \\
Pore volume $(\mathrm{mL})$ & 1062 \\
Permeability $\left(\mu \mathrm{m}^{2}\right)$ & 3.35 \\
Confining pressure used $(\mathrm{MPa})$ & 14.0 \\
\hline
\end{tabular}

Table 6.2: Properties of the porous medium.

\begin{tabular}{lcccc}
\hline Oil & Density $(\mathrm{g} / \mathrm{cc})$ & Viscosity (Poise) & $\begin{array}{c}\text { Core Average } \\
\phi \text { at maximum } \\
\text { drawdown }\end{array}$ & $\beta$ \\
\hline Lloydminster & 0.968 & 30.07 & 0.138 & 3.40 \\
Lindbergh & 0.978 & 39.70 & 0.148 & 3.17 \\
\hline
\end{tabular}

Table 6.3: Properties of "live oil" at saturation.

transducers were placed at intervals along the pack. Each transducer measures the pressure drop between two taps equally spaced along the pack; the pressure drop across two taps is called a "differential pressure." The plots given in figures 6.2 and 6.3 are of straight line segments between pressure taps. Maini \& Sarma [1994] also did "blowdown" experiments in which the inlet was closed and pressure at the outlet suddenly dropped to $0.1 \mathrm{MPa}$ (see figure 9.1). 


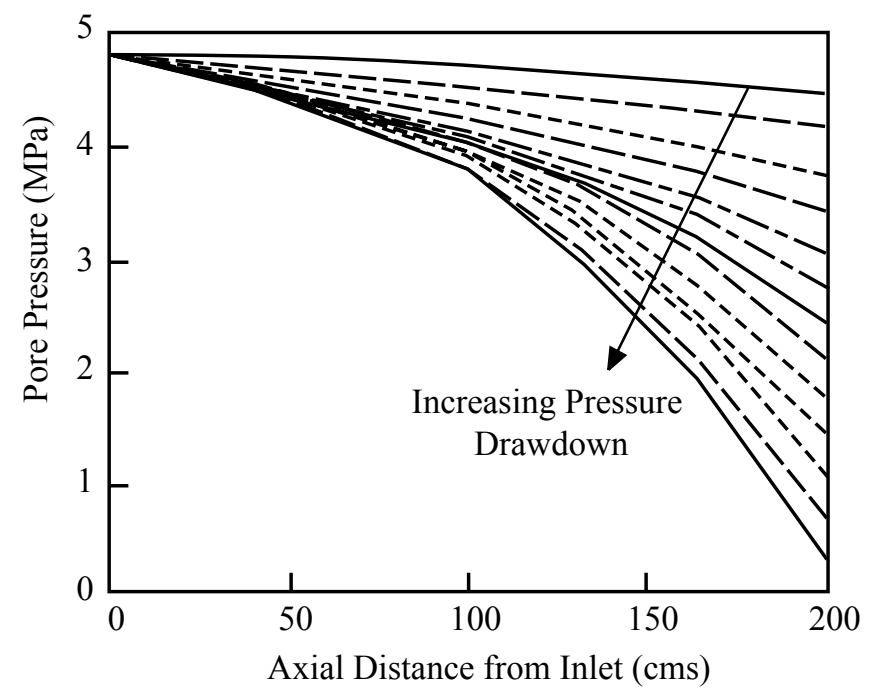

Figure 6.2: (Maini \& Sarma, 1994). Pressure distributions in steady flows of Lindbergh oils at various pressure drawdowns.

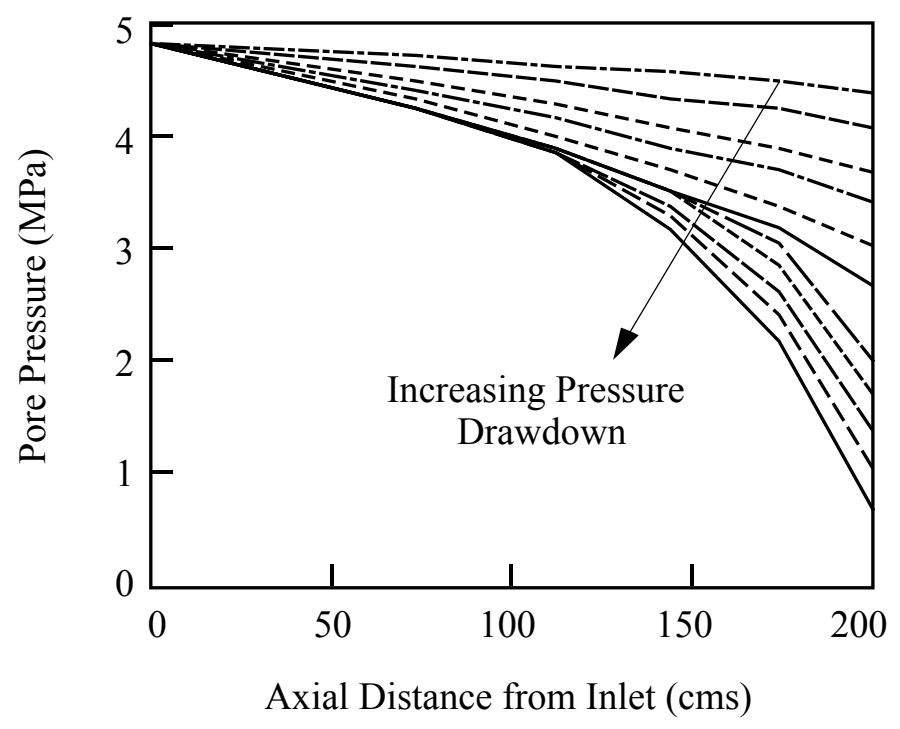

Figure 6.3: (Maini \& Sarma, 1994). Pressure distributions in steady flow of Lloydminster oils at various pressure drawdowns. 


\section{Dimensionless equations, rate constant and viscosity func- tion}

We are going to choose model parameters to get a best fit to the experiments of Maini and Sarma [1994]. To get the maximum generality from our study and to identify the fitting parameters it is useful to express the governing equations (4.27) and (4.28) in dimensionless form. We introduce dimensionless variables

$$
\left.\begin{array}{rl}
X & =x / L \\
T & =t / \theta \\
P & =p / \tilde{p} \\
\Lambda(\phi) & =\lambda(\phi) / \lambda_{0}=\eta(0) / \eta(\phi)
\end{array}\right\}
$$

and find that if

$$
\theta=\alpha L^{2} / \lambda(0) \tilde{p}
$$

then

$$
\frac{\partial \phi}{\partial T}=\frac{\partial}{\partial X}\left[(1-\phi) \Lambda(\phi) \frac{\partial P}{\partial X}\right]
$$

and

$$
\frac{J}{(1-\phi)^{2}}\left\{\frac{\partial \phi}{\partial T}-\Lambda(\phi) \frac{\partial P}{\partial X} \frac{\partial \phi}{\partial X}\right\}=\frac{1-P}{P}-\frac{\beta \phi}{1-\phi}
$$

where $L$ is the pack length and

$$
J=\tau \tilde{p} \lambda(0) / L^{2}
$$

is the dimensionless relaxation parameter. The outlet is at $X=0$ and the inlet at $X=1$. The system (7.3) and (7.4) is completely determined by a rate constant $J$ and a permeability function $\Lambda(\phi)$ or equivalent viscosity function $\eta(\phi)$.

We are dealing with dispersions of small gas bubbles that are spherical when

not crowded. It is usual to fit the viscosity function of equivalent dispersions of solid spheres with

$$
\frac{\eta(\phi)}{\eta(0)}=\left(1-\frac{\phi}{A}\right)^{-m}
$$


where $A$ is a maximum packing fraction, $\phi \leq A$, and $m>0$ is selected from data; $m \approx 2$ works well.

It is altogether different for gas bubbles at large $\phi$ where stabilized bubbles would change phase, first to wet and then to dry foams. To be consistent with the solubility expression (2.14) we should seek a viscosity that increases continually with $\phi$ from dilute dispersions with $\phi \approx 0$ to dry foam with $\phi \approx 1$; hence $A=1$ and $m$ is to be determined. The viscosity function

$$
\eta(\phi)=\frac{\eta(0)}{(1-\phi)^{m}}, m>0
$$

says that dry foam has an infinite resistance to flow. Of course, the foam would break under pressure for dispersed fraction $\phi$ near one; the gas bubbles would collapse with free gas channels percolating through the pack. We have a one parameter family of viscosity functions depending on the exponent $m$. After fitting our model to the experimental data of Maini and Sarma [1994] we found that $m=11$, which at small $\phi$ gives

$$
\eta(\phi)=\eta(0)(1+11 \phi+\cdots) .
$$

The value 11 is greater than the Einstein value $m=2.5$ for dilute dispersions of solid spheres possibly because of the additional increases in the viscosity of the liquid phase caused by the vaporization of dissolved gases; in other words, the viscosity of mixture increases because there is less dissolved and more dispersed gas.

It follows now that the system (7.3) and (7.4) is well characterized when the parameter $J$ and $m$

$$
\Lambda(\phi)=(1-\phi)^{m}
$$

are known. We have tried to fit the model predictions to the experiments with a nearly best, but imperfect fit, when

$$
m=11, \quad J=5 .
$$

In the next section we describe the experiments and compare the data with the model prediction. Using the data given in section 6 we found that

$$
J=1.345 \times 10^{-6} \tau .
$$

Our data fitting gives $J=5$ or $\tau=3.71 \times 10^{6} \mathrm{sec}$. 


\section{Steady flow in a horizontal sandpack}

Foamy oil is driven from a reservoir at saturation $P=1$ at $X=0$ to another reservoir at the outlet pressure $p_{L}, P_{L}=p_{L} / \tilde{p}<1$. Since $\partial \phi / \partial T=0$ for steady flow, equation (7.3) implies that

$$
-\Lambda(\phi)(1-\phi) \frac{d P}{d X}=C=\text { constant }
$$

Equation (8.1) says that the oil velocity

$$
-\lambda \frac{d P}{d X}(1-\phi)
$$

is a constant in steady flow, independent of $X$. Combining (8.1) and (7.4) we get

$$
\frac{J C}{(1-\phi)^{3}} \frac{\partial \phi}{\partial X}=\frac{1-P}{P}-\frac{\beta \phi}{1-\phi}
$$

Equations (8.1) and (8.3) are to be solved subject to the conditions that

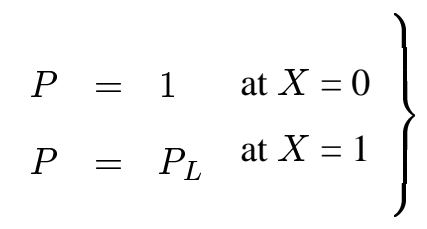

when $J=0$

$$
P=\frac{1-\phi}{(\beta-1) \phi+1}, \frac{d P}{d X}=\frac{-\beta d \phi / d X}{[(\beta-1) \phi+1]^{2}}
$$

and

$$
\left.\begin{array}{ll}
\phi=0 & \text { at } X=0 \\
\phi=\phi_{L}=\frac{1-P_{L}}{1+(\beta-1) P_{L}} & \text { at } X=1
\end{array}\right\}
$$

Elimination of $d P / d X$ in (8.1) then gives rise to the following quadrature 


$$
C X=\int_{0}^{\phi} \frac{\beta \Lambda(\eta)(1-\eta) d \eta}{[1+(\beta-1) \eta]^{2}}
$$

The constant $C$ is then determined by the outlet pressure given by (8.6). The integral in (8.7) can be done when $\Lambda(\eta)$ is given by (7.8).

In general, when $J=0$, the first order system (8.1) and (8.3) may be solved numerically. The steady distributions of pressures along the sandpack are compared with data from experiments in figures 8.1-8.3.

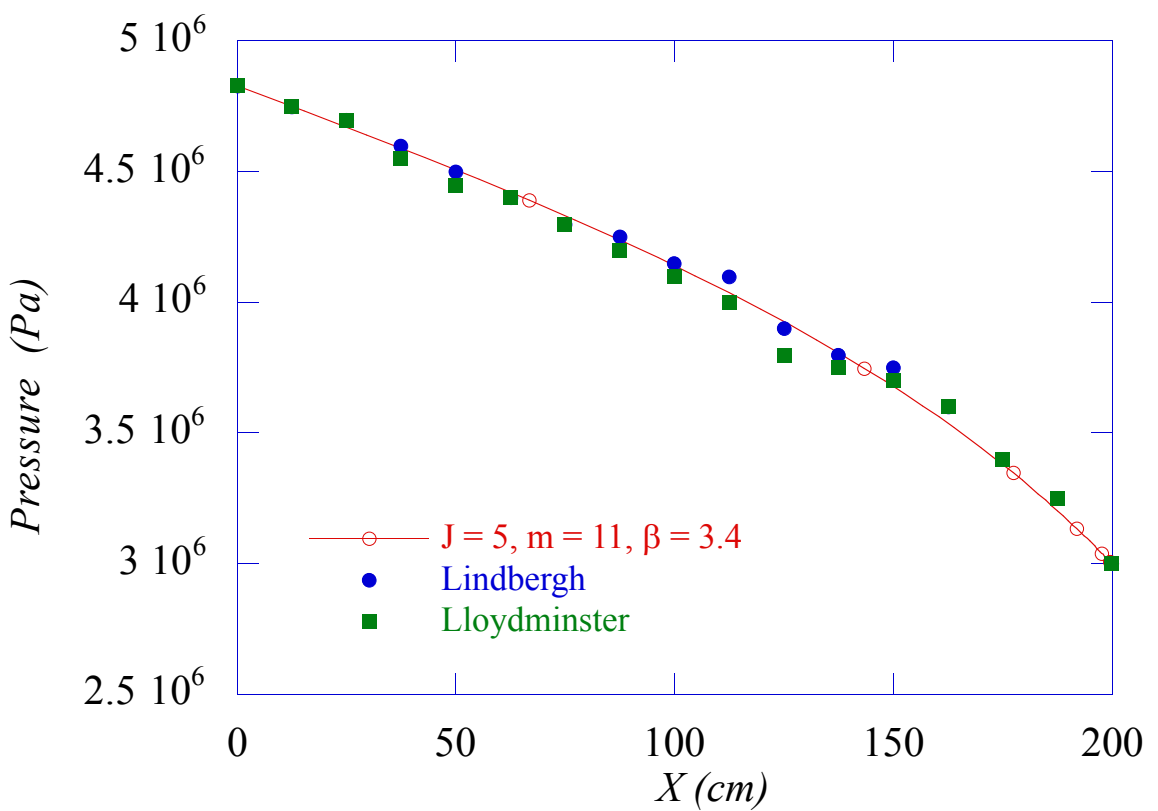

Figure 8.1: Comparison of theoretical and experimental pressure distributions at drawdown pressure $p_{l}=3 \mathrm{MPa}$. 


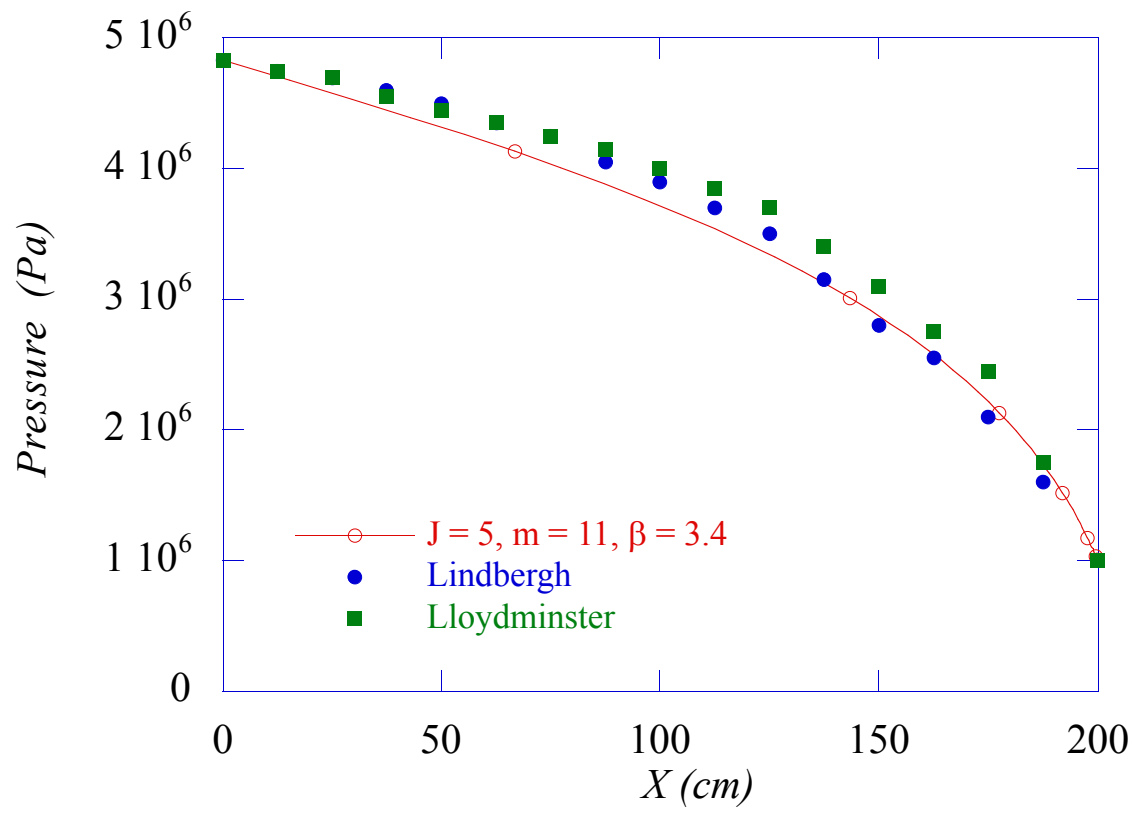

Figure 8.2: Comparison of theoretical and experimental pressure distributions at drawdown pressure $p_{l}=1 \mathrm{MPa}$. 


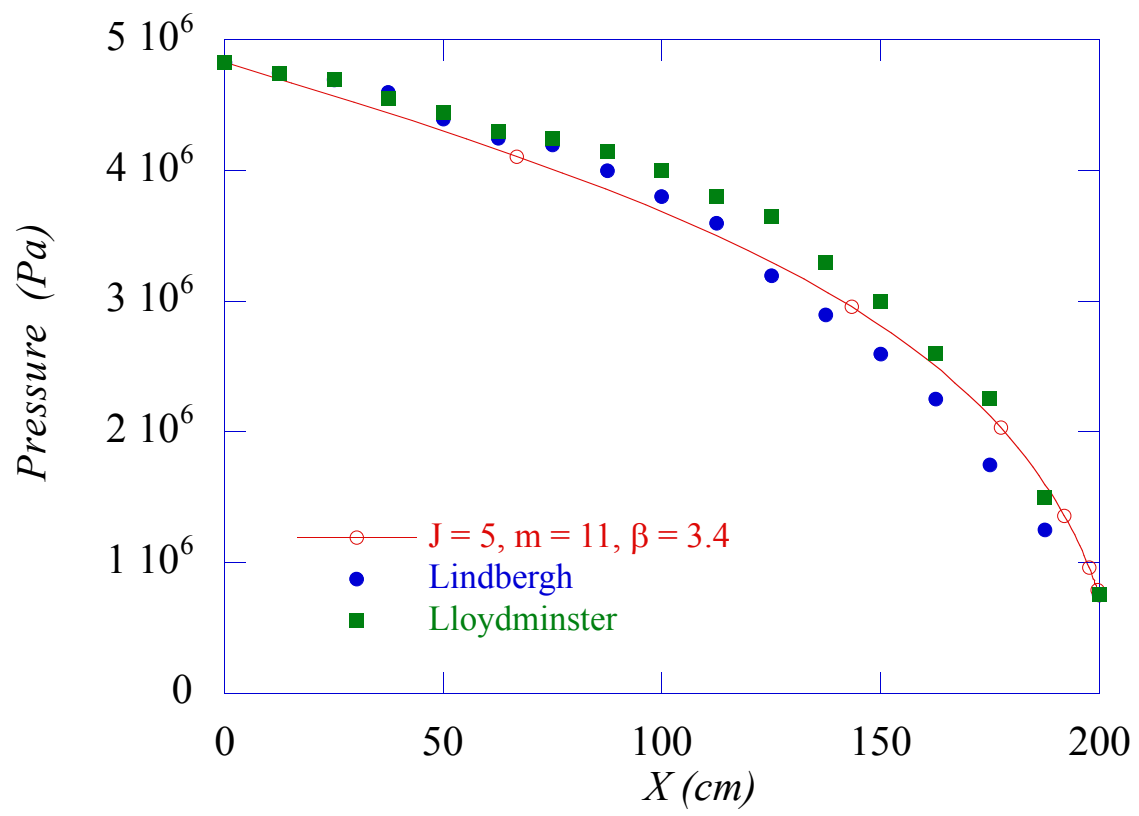

Figure 8.3: Comparison of theoretical and experimental pressure distributions at drawdown pressure $p_{l}=0.75 \mathrm{MPa}$. 


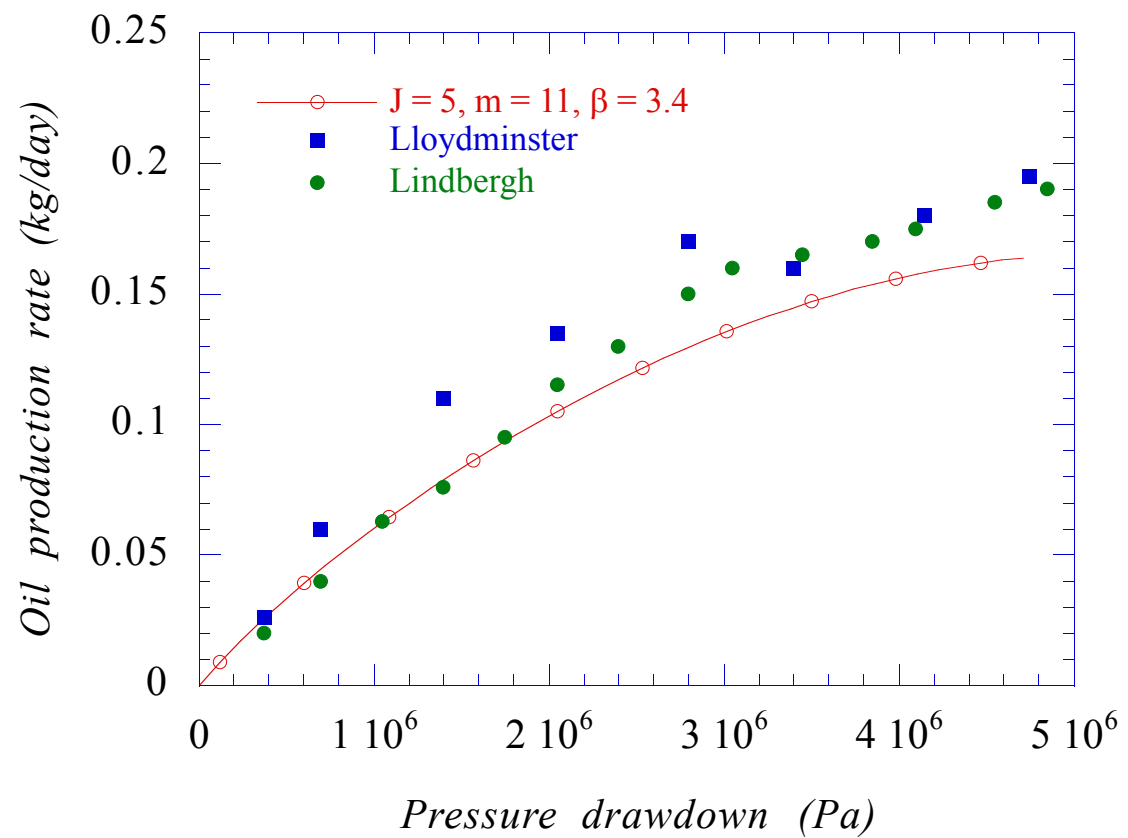

Figure 8.4: Comparisons of oil production rates in steady flows at various pressure drawdowns. 
Maini and Sarma [1994] also reported the rate of oil production in steady state as a function of the drawdown pressure. The oil production is the mass flow rate, the volume flow rate of oil times the oil density $\rho \approx 0.97 \mathrm{~g} / \mathrm{cc}$. The superficial mixture velocity $\mathbf{u}$ is given by Darcy's law (4.16), $A \mathbf{u}$, where $A$ is the cross-sectional area, is the volume flow rate of the mixture of gas and oil, which is larger than the volume flow rate $\mathbf{u}(1-\phi)$ of oil alone. Hence the mass flow rate of oil alone is

$$
\rho_{l} \stackrel{\circ}{Q}=-\rho_{l} A(1-\phi) \lambda(\phi) \frac{d P}{d X}
$$

which according to (8.2) is a constant, independent of $X$. A comparison of the measured and theoretical results for $m=11, J=5, \beta=3.4, A=16.1 \times 10^{-4} m^{2}$ is given in figure 8.4.

The modest discrepancy between measured and theoretical values for large drawdowns may be due to the formation of foam. This foaming might be expected to reduce the rate of production because of flow resistance, and to increase the primary recovery because foam effectively traps the gas; all the oil must come out with trapped gas.

\section{Blowdown experiment}

We turn next to the blow down experiment of Maini and Sarma [1994] (their figure 9, our figure 9.1). They describe their experiment as follows (the emphasis indicated is ours).

A different type of experiment was needed to estimate the total recovery potential of solution gas drive. This experiment started with the sand pack at maximum "live oil" saturation. The pack was allowed to blow down to atmospheric pressure through the outlet end, and the inlet end remained closed. Figure 9 shows the recovery and pressure-drop behavior. More than 20\% of the original oil was recovered in this primary depletion experiment. The value is surprisingly high for the viscous oil system and suggests that the critical gas saturation was much higher than what would be measured by an external gas drive experiment. Typically, the external drive experiments in such systems show the critical gas saturation to be less than 5\%. Therefore, this experiment also suggests that a mechanism is present in heavy-oil systems to increase the critical gas saturation. We suggest that this mechanism is the formation of an oil-continuous foam. 
We have already remarked that the solubility isotherm with $\beta=3.4$ or 3.17 leads to dispersed gas fractions of the order 0.93; foaming is inevitable.

The blowdown experiment is unsteady. The experiments shown in figure 9.1 show that the pack is still producing oil after 200 hours; the terminal steady and uniform state has not been achieved.

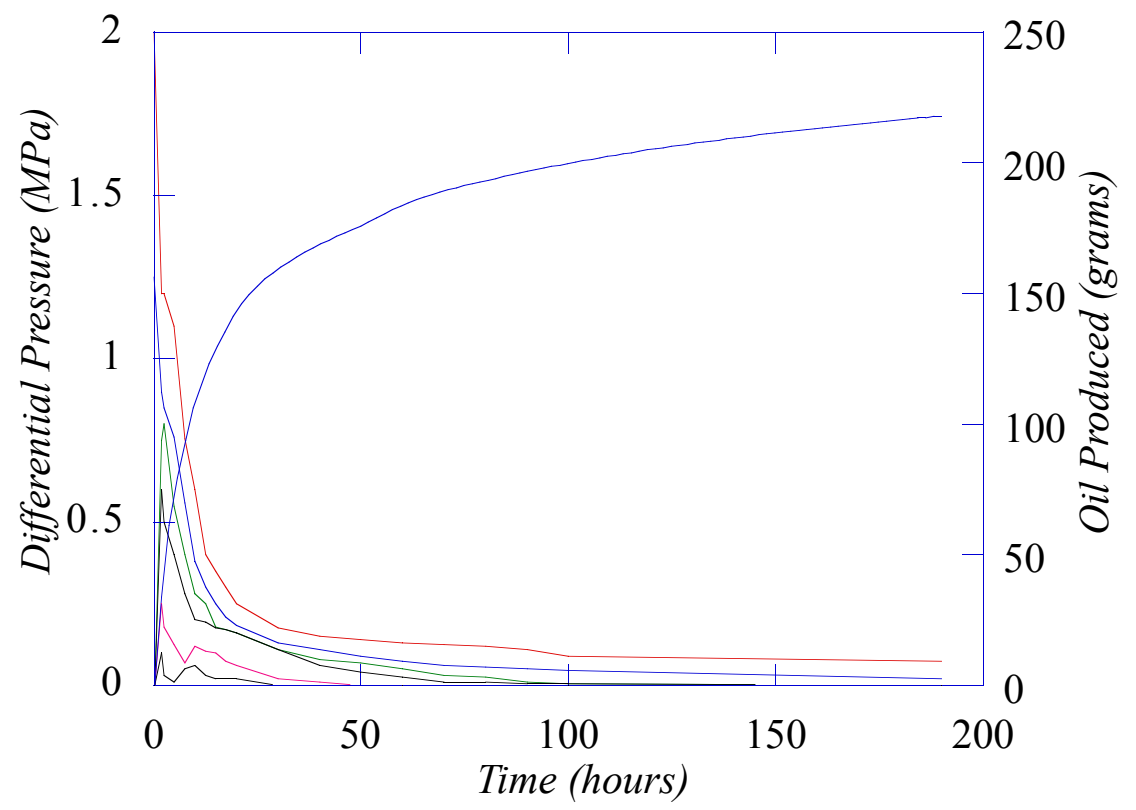

Figure 9.1: (Maini \& Sarma, 1994). Change in the pressure drop across different core segments and cumulative oil production with time during the blowdown experiment with the Lloydminster system.

The initial value problem corresponding to the blowdown problem is to solve (7.3) and (7.4) subject to the initial conditions

$$
P=1, \quad \phi=0 \quad \text { for } 0 \leq X \leq 1 \text { when } T \leq 0
$$

and boundary conditions

$$
\frac{d P}{d X}=0 \quad \text { at } X=1
$$

and 


$$
P=P_{L}[1-H(t)] \quad \text { at } X=0
$$

where $H(t)$ is unit step function $p_{L}=0.1 \mathrm{MPa}, \tilde{p}=4.83 \mathrm{MPa}, P_{L}=p_{L} / \tilde{p}=$ 0.0205 . The integration of these equations was carried by numerical methods.

The cumulative production may be obtained as the integral over time of the rate of production

$$
\rho_{o} Q(t)=\rho_{o} \int_{o}^{t} \stackrel{\circ}{Q}(t) d t
$$

where

$$
\stackrel{\circ}{Q}=A \lambda(1-\phi) d P / d X
$$

is evaluated at $X=0$, the open end of the sandpack. A comparison of the model predition with $m=11$ and $J=5$ and the experiment are shown in figure 9.2. The values $m=11, J=5$ were selected for a best fit for the steady pressure distributions given in figure 8.1 through 8.3 , the rate of production in steady flow given in figure 8.4 and the comparison of theory and experiment in drawdown given in figure 9.2 . 


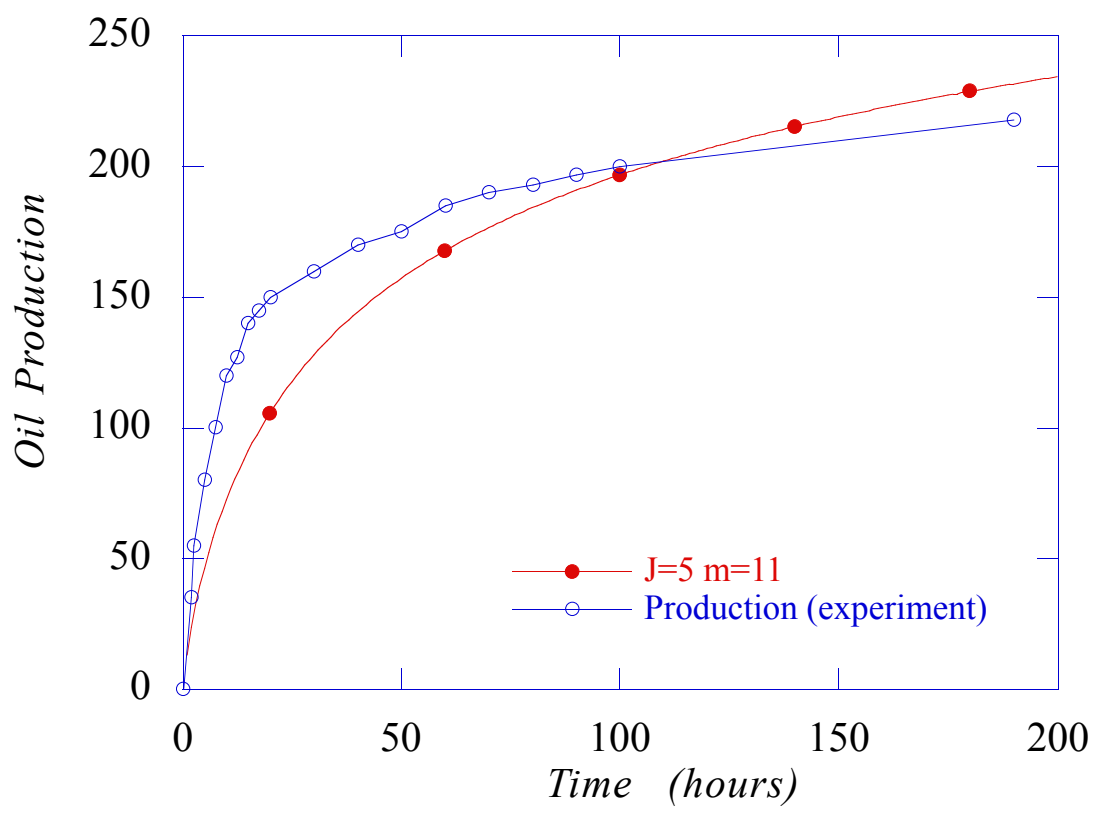

Figure 9.2: Comparison of theory and drawdown of pressure in experiment for Lloydminster oil. 


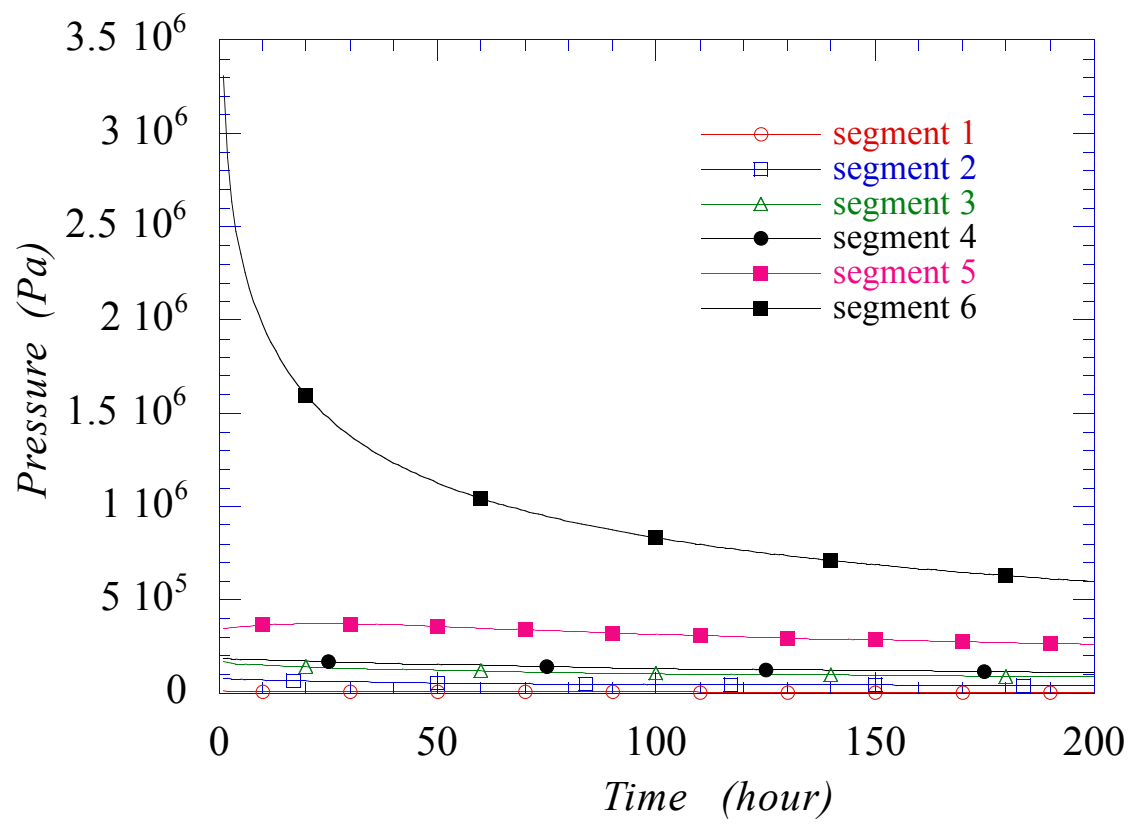

Figure 9.3: Model prediction of the evolution of the differential pressure in 6 segments for the blowdown experiment in figure 9.1. $\beta=3.4, m=11, J=5$.

The differential pressure in the 6 segments defined by pressure tabs is given in figure 9.3. The theory greatly overpredicts the drop in pressure in drawdown.

The blowdown experiment produces large amounts of gas because the pressure drop is very severe. The appearace of foam was observed, consistent with rapid outgassing. Blowdown also favors the formation of connected gas, due to bubble rupture, which percolates out of the sandpack without a trace reducing both the pressure and the emulative production. These effects of free gas are not allowed in our model and may account for the discrepancies between theory and experiment exhibited in figures 9.2 and 9.3.

\section{Discussion and Conclusion}

The theory which we have developed could be called a continuum mixture theory for foamy oils flow with dispersed gas of low mobility relative to the liquid which leads to three coupled nonlinear partial differential equations for $\mathbf{u}, p$ and the gas fraction $\phi$, five scalar equations in five unknowns. 
We derived an equation of state (called an equilibrium isotherm) for foamy oil. This equation gives the volume fraction of dispersed gas at a given temperature and pressure at equilibrium for the situation in which the gas that comes from releasing disolved gas remains dispersed. This equation depends on only one material coefficient, a function of the temperature which can be obtained from measurement in a PVT cell. Such measurements were made and yielded only modestly different values of the coefficient for two Canadian and one Venezuelan crude oils. The empirically based equation of state avoids constitutive hypotheses about nucleation and bubble growth, which are implicit in the measured equilibrium values of the volume fraction of dispersed gas. The derivation and application of equilibrium isotherms is one of the principal contributions of this paper.

Another contribution is the development of a flow description for foamy oil whose center pieces are a continuity equation for the bubbly mixture, Darcy's law and a relaxation equation for the equilibrium isotherm. The relaxation equation depends on a to-be-determined rate constant which allows for delay in the growth of the gas fraction $\phi$ to a change of pressure expressing the idea that it takes time for bubbles to nucleate and grow. It is possible that the pressure also relaxes, but the modeling of pressure relaxation would introduce another rate constant and is left for future work.

The mobility function $\lambda(\phi)$ which appears in Darcy's law must also be modeled. The mobility function for our model is the measured permeability of the porous media over the to-be-determined effective viscosity of the mixture.

The modeling of the viscosity of the mixture as a function of the dispersed gas is very difficult and we have no guidelines from the literature. Our model, which depends only on the dispersed gas fraction, makes no formal distinction between bubbly mixture and the foam that arises inevitably under the kind of severe depressurization seen in the Maini-Sarma blowdown experiments. The range of the dispersed gas fraction is from $\phi=0$, in which the oil is saturated with dissolved gases, to $\phi=1$ which can be interpreted as a super-dry foam. The viscosity of the foam ought to be much greater than the bubbly mixture but less great than concentrated dispersions of solids that can't move at all when they are closely packed. An additional complication in the modeling of the viscosity is that the viscosity of the liquid increases rather sharply when gas is released; its like lowering the fraction of water in a glycerin and water mixture. So the viscosity of the mixture increases both because of the presence of more bubbles and because the continuous liquid-phase viscosity also increases.

We related our flow description to the conventional description of two-phase flow in porous media using the concept of relative permeability. We write down all the usual equations with the caveat that the mobility function for each phase is expressed in terms of an effective viscosity rather than a relative permeability; for 
the gas we have

$$
\lambda_{g}(\phi)=\frac{k k_{g}}{\eta_{g}}=\frac{k}{\mu_{g}(\phi)}
$$

where $k$ is permeability, $k_{g}$ is relative permeability, $\eta_{g}$ is gas viscosity, and $\mu_{g}(\phi)$ is effective gas viscosity. Of course, we have done nothing but change the names of symbols, but our concept is closer to mixture theory in which we describe flow properties of fluid phases in a porous media of fixed properties independent of the fluid and flow. Moreover, our effective viscosities can be directly related to the measured values of the mixture viscosity

$$
\mu(\phi)=\left(\frac{1}{\mu_{g}(\phi)}+\frac{1}{\mu_{l}(\phi)}\right)^{-1}
$$

where $\mu_{l}(\phi)$ is the effective viscosity of the liquid phase.

Our mixture theory arises as a rigorous consequence of the conventional theory under the assumption that the average velocities of dispersed gas and liquid are identical and in this case we get explicit

$$
\begin{aligned}
\mu_{g}(\phi) & =\frac{\mu(\phi)}{\phi} \\
\mu_{l}(\phi) & =\frac{\mu(\phi)}{(1-\phi)}
\end{aligned}
$$

relating effective viscosity of the phases to the effective viscosity of the mixture. In addition we find that

$$
\frac{k_{r l}}{k_{r g}}=\frac{1-\phi}{\phi} \frac{\eta_{l}}{\eta_{g}}
$$

relating the ratio of relative permeabilities to the ratio of molecular viscosities.

We applied our theory to the sandpack experiments of Maini \& Sarma [1994], backing out the rate constant and mixture viscosity function to fit data. The viscosity function was selected in the frequently used form

$$
\mu(\phi)=\mu(0) /\left(1-\frac{\phi}{\phi_{m}}\right)^{m}
$$


where $\phi_{m}$ is the maximum packing fraction. $\phi_{m}$ is a perfectly clear concept for solid spheres and might be considered for bubbly mixtures. But if our bubbly mixtures foam we can get dispersed gas all the way to $\phi=1$, hence $\phi_{m}=1$. Our equilibrium isotherm also allows for a full range of dispersed gas fractions $0 \leq \phi \leq 1$ up to dry foams. We then look for a single continuous expression for $\mu(\phi)$ in the form (10.6) with $\phi_{m}=1$. We think that dry foams are rigid unless riding on a liquid layer, which is not easily formed in a porous media; hence $\mu(\phi) \rightarrow \infty$ as $\phi \rightarrow 1$.

In the dilute limit

$$
\mu(\phi) \rightarrow \mu(0)(1+m \phi) .
$$

In foamy oil $m$ should be larger than the Einstein value 2.5 because of the added viscosity increase of the continuous oil phase due to the release of dissolved gas.

We backed out a viscosity function by fitting, finding $m=11$ together with a relaxation time corresponding to a dimensionless time $J=5$. With these values we achieved some good agreements between the sandpack model prediction and data for steady flow but with larger discrepancies in blowdown experiments in which dispersed gas probably percolated into free gas.

We think that this comparison of model and experiment establishes a direction for model building using the average velocity of phases, the pressure and volume fraction of dispersed gas as primitive variables. It is possible for the dispersed gas to move relative to the oil without forming connected gas through coalescence and percolation. To describe such a condition we would need to consider momentum and mass balances of the two phases, but the description could be formed with same set of primitive variables, replacing constitutive equations for nucleation, bubble growth and possibly some coalescence, with relaxation of equilibrium isotherms from PVT data.

Our model has limitations; it only applies for small bubbles, but not too small either. Since monitoring of bubble size in porous medium is hard to do, or even presently impossible, there is no way of knowing whether the assumptions that we made are valid. The results seem to indicate that they might be. Models, like the one proposed here, should be tested against more data particularly against data from producing fields. For production the conditions for the breakdown of models that work when all the gas is dispersed due to the formation of connected gas is even more important than the models themselves. 
Acknowledgement. The work of D.D. Joseph was supported in part by the DOE (Engineering Research Program of the Department of Basic Energy Sciences), the NSF under Grant Opportunities for Academic Liasons with Industry and the Minnesota Supercomputer Institute. We are indebted to Prof. G.I. Barenblatt for providing us with a translation of the prescient work of Leibenson [1941] and for calling our and the reader's attention to the possibility that gas release in reservoirs could be quite different than in a PVT cell or even in a sandpack.

\section{References}

1. Claridge, E.L. and Prats, M. June 19-21, 1995. "A Proposed Model and Mechanism for Anomalous Foamy Heavy Oil Behavior," paper SPE 29243 presented at the International Heavy Oil Symposium, Calgary, AB Proc., 9-20; also the unsolicited manuscript of SPE (USMS) 29243, 1994.

2. Firoozabadi, A., Ottesen, B., \& Mikkelsen, M. December 1992. "Measurement of Supersaturation and Critical Gas Saturation”, SPE Formation Evaluation, 337-344.

3. Huerta, M., Otero, C., Rico, A., Jiménez, I., De Mirabal, M. \& Rojas, G., October 6-9, 1996. "Understanding Foamy Oil Mechanisms for Heavy Oil Reservoirs during Primary Production”, paper SPE 36749, presented at the 1996 SPE Annual Technical Conference and Exhibition, Denver, Colorado, 671-685.

4. Joseph, D.D., A.M. Kamp and R. Bai 2002. "Foamy Oil Flow in Porous Media," Confinement and Remediation of Environmental Hazards and Resource Recovery, Inst. Math. Applications, Vol \# J. Chadam, A. Cunningham, R.E. Ewing, P. Ortoleva, M. Wheeler, Eds. Springer-Verlag. To appear.

5. Joseph, D.D. and Y. Renardy 1992. Fundamentals of Two-Fluid Dynamics, Vol. I: Mathematical Theory and Applications, Vol. II: Lubricated Transport, Drops and Miscible Liquids, Springer.

6. Kraus, W.P., McCaffrey, W.J., \& Boyd, G.W. 1993. "Pseudo-Bubble Point Model for Foamy Oils," paper CIM 93-94 presented at the 44th Annual Technical Conference of the Petroleum Society of CIM, Calgary, AB, May 9-12.

7. Lebel, J.P. March 2, 1994. "Performance implications of various reservoir access geometrics", Paper presented at the $11^{\text {th }}$ Annual Heavy Oil \& Oil Sands Tech. Symp..

8. Leibenson, L.S., 1941. "The motion of gas-saturated fluid in a porous media. Bulletin," USSR Acad. Science, Ser. Geography \& Geophysics, No. 3.

9. Maini, B.B., June 1996. "Foamy Oil Flow in Heavy Oil Production," JCPT 35(6), 21-24.

10. Maini B.B. \& Sarma, H. 1994. "Role of Nonpolar Foams in Production of Heavy Oils," in: "Advances in Chemistry Series" 242: 405-420. 
11. De Mirabal, M., Gordillo, R., Fuenmayor, M., Rojas, G., Rodriguez H. \& Sanchez, R. April 23-26, 1996. "Integrated Study for the Characterization and Development of the MFB-53 Reservoir, North Hamaca-Orinoco Belt, Venezuela", paper SPE 36095, presented at the Fourth Latin American \& Caribbean Petroleum Engineering Conference, Port-of-Spain, Trinidad \& Tobago.

12. Mastmann, M., Moustakis, M. \& Bennion, D.B. 2001. "Predicing foamy oil recovery," SPE 68860, SPE Western Regional meeting held in Bakersfield, CA, 26-30 March 2001.

13. Peng, D.Y., Fu, C.T., Bird, G.W. \& Hsi, C., August, 4-9, 1991. "Effect of Gas components on thermodynamic properties of Alberta Heavy Crudes and Bitumens," in: 5th Unitar Heavy Crude \& Tar Sands International Conference, Caracas, Venezuela, Proc. 1: 47-55.

14. Pooladi-Darvish M. \& Firoozabadi, A. June 11, 1997. "Solution gas drive in heavy oil reservoirs", paper no. 97-113, presented at the 48th Annual Technical meeting of the Petroleum Society of CIM in Calgary, Canada.

15. Sheng, J.J., Hayes, R.E., Maini A.B. \& Tortike, W.S. 1996. "A dynamic model to simulate foamy oil flow in porous media," SPE 36750.

16. Sheng, J.J., Maini, B.B., Hayes R.E. \& Tortike, W.S. May 1999a. "Critical Review of foamy Oil Flow", Transport in Porous Media, 35(2), 157-187.

17. Sheng, J.J., Hayes, R.E., Maini, B.B. \& Tortike, W.S. May 1999b. "Modeling Foamy Oil Flow in Porous Media", Transport in Porous Media, 35(2), 227-258.

18. Svrcek, W.Y. \& Mehrotra, A.K. 1982. "Gas solubility, viscosity and density measurements for Athabasca bitumen,” J. Canadian Petroleum Technology, 21(4), 31-38. 